\title{
RATIO LIMIT THEOREMS FOR RANDOM WALKS ON GROUPS $\left({ }^{1}\right)$
}

\author{
BY \\ CHARLES STONE
}

I. Introduction and statement of results. In this paper we will obtain ratio limit theorems for random walks on locally compact Abelian groups.

Let $G$ be a locally compact Abelian group, let $e$ denote the identity of $G$, and let | | denote Haar measure on $G$.

Let $\mu$ denote a regular probability measure on $G$, and let $\mu^{(n)}$ denote the $n$-fold convolution of $\mu$ with itself. Let $S$ denote the support of $\mu$ defined by

$$
S=\{x \in G \mid \mu(P)>0 \text { whenever } P \text { is an open nbhd. of } x\} .
$$

Then $S$ is closed and $\mu(S)=1$. Throughout this paper we make the

Basic Assumption. The closed subgroup of $G$ generated by $S-S$ is $G$ itself.

This assumption involves no essential loss of generality since for the results considered here the general case, when properly formulated, can be reduced easily to this special case.

In order to prove our first theorem we will need a result concerning Haar measure on $G$. Since this result, which depends on the structure theory for $G$, seems to be new and of independent interest, we state it here as

Proposition 1. Let $A$ be a compact set and $B$ be an open set, both having finite positive Haar measure. Then for every $\varepsilon>0$ there is an open set $P$, a compact set $D \subset P$, integers $m$ and $k$ and points $x_{1}, \ldots, x_{m}, y_{1}, \ldots, y_{k}$ in $G$ such that

$$
m|B| \leqq(1+\varepsilon) k|A|, \quad \bigcup_{1 \leqq j \leqq m} x_{j}+D \supset A
$$

and $y_{j}+P, 1 \leqq j \leqq k$, are disjoint subsets of $B$.

Next we will prove

THEOREM 1. Let $A$ be a compact set and $B$ an open set, both having finite positive Haar measure. Then for any integer $n_{0}, \varepsilon>0$, and compact set $C$, there is $a \delta>0$ such that for $n$ sufficiently large

$$
\mu^{\left(n+n_{0}\right)}(x+y+A) /|A| \leqq(1+\varepsilon) \mu^{(n)}(x+B) /|B|+e^{-\delta n}, \quad x \in G \text { and } y \in C .
$$

Received by the editors January 10, 1966 and, in revised form, May 12, 1966.

(1) The preparation of this paper was supported in part by NSF Grant GP-5224. 
Theorem 1 implies that ratio limits exist if $\mu$ satisfies

Condition 1. There is a compact set $C$ such that $\lim _{\sup } \rightarrow \infty\left(\mu^{(n)}(C)\right)^{1 / n}=1$.

Let $\mathscr{A}$ denote the collection of all Borel sets $A$ such that $A$ is relatively compact and $0=|\partial A|<|A|$. Then we have

Corollary 1. Suppose Condition 1 holds. Then for $A$ and $B$ in $\mathscr{A}$ and $n_{0}$ an integer

$$
\lim _{n \rightarrow \infty} \frac{\mu^{\left(n+n_{0}\right)}(x+A)}{\mu^{(n)}(y+B)}=\frac{|A|}{|B|}
$$

uniformly for $x$ and $y$ in compact sets.

We call $\mu$ symmetric if $\mu(-A)=\mu(A)$ for all Borel sets $A$.

Proposition 2. If $\mu$ is symmetric, then Condition 1 holds.

Let $\mathscr{S}$ denote the collection of continuous homomorphisms from $G$ to $R$. Let $g$ denote the extended real-valued function defined on $\mathscr{S}$ by

$$
g(s)=\int_{G} e^{s(x)} \mu(d x), \quad s \in \mathscr{S}
$$

Proposition 3. If $g(s)<1$ for some $s \in \mathscr{S}$, then Condition 1 doesn't hold.

Proposition 4. Suppose $G$ is compactly generated. Then Condition 1 holds if and only if $\mathscr{S}(s) \geqq 1$ for all $s \in \mathscr{S}$.

Even if Condition 1 doesn't hold we can still obtain ratio limit theorems provided that $G$ is compactly generated and $\mu$ satisfies

Condition 2. The smallest closed semigroup containing $S$ in $G$ itself.

COROLlaRY 2. Suppose $G$ is compactly generated and Condition 2 holds. Then there is a unique $s_{0} \in \mathscr{S}$ such that $g\left(s_{0}\right)=\inf _{s \in \mathscr{S}} g(s)$. Moreover for $A$ and $B$ in $\mathscr{A}$

$$
\lim _{n \rightarrow \infty} \frac{\mu^{\left(n+n_{0}\right)}(x+A)}{\mu^{(n)}(y+B)}=\left(g\left(s_{0}\right)\right)^{n_{0}} \exp \left(s_{0}(y-x)\right) \frac{\int_{A} \exp \left(-s_{0}(z)\right) d z}{\int_{B} \exp \left(-s_{0}(z)\right) d z}
$$

uniformly for $x$ and $y$ in compact sets.

Let $p^{(n)}$ denote the Radon-Nikodym derivative of $\mu^{(n)}$ with respect to Haar measure. (It follows from our basic assumption on $\mu$ that $G$ must be $\sigma$-compact and 
hence that the Haar measure on $G$ is $\sigma$-finite. Thus $p^{(n)}$ is well defined.) If $p^{(n)}$ can be chosen to be continuous or bounded we assume that this has been done.

The above results can be sharpened if $\mu$ satisfies

Condition 3. For some $n \mu^{(n)}$ is absolutely continuous with respect to Haar measure and has a bounded density $p^{(n)}$.

THEOREM 2. Suppose Condition 3 holds. For any compact set $C$, integer $n_{0}$ and $\varepsilon>0$, there is $a \delta>0$ such that for $n$ sufficiently large

$$
p^{\left(n+n_{0}\right)}(x+y) \leqq(1+\varepsilon) p^{(n)}(x)+e^{-\delta n}, \quad x \in G \text { and } y \in C .
$$

Corollary 3. Suppose Conditions 1 and 3 hold. Then for any integer $n_{0}$

$$
\lim _{n \rightarrow \infty} \frac{p^{\left(n+n_{0}\right)}(x)}{p^{(n)}(y)}=1
$$

uniformly for $x$ and $y$ in compact sets.

Corollary 4. Suppose $G$ is compactly generated and Conditions 2 and 3 hold. Let $s_{0}$ be as in Corollary 2. Then

$$
\lim _{n \rightarrow \infty} \frac{p^{\left(n+n_{0}\right)}(x)}{p^{(n)}(y)}=\left(g\left(s_{0}\right)\right)^{n_{0}} \exp \left(s_{0}(y-x)\right)
$$

uniformly for $x$ and $y$ in compact sets.

Interesting results also follow if $\mu$ satisfies

Condition 4. For some $n, \mu^{(n)}$ has a nontrivial absolutely continuous component with respect to Haar measure.

Let $\mathscr{B}$ denote the collection of all Borel sets $B$ such that $B$ has compact closure and positive Haar measure.

Theorem 3. Suppose Condition 4 holds. Let $A$ and $B$ be in $\mathscr{B}$. Then for any integer $n_{0}, \varepsilon>0$, and compact set $C$, there is $a \delta>0$ such that for $n$ sufficiently large

$$
\mu^{\left(n+n_{0}\right)}(x+y+A) /|A| \leqq(1+\varepsilon) \mu^{(n)}(x+B) /|B|+e^{-\delta n}, \quad x \in G \text { and } y \in C .
$$

Corollary 5. Suppose Conditions 1 and 4 hold. Let $A$ and $B$ be in $\mathscr{B}$. Then

$$
\lim _{n \rightarrow \infty} \frac{\mu^{\left(n+n_{0}\right)}(x+A)}{\mu^{(n)}(y+B)}=\frac{|A|}{|B|}
$$

uniformly for $x$ and $y$ in compact sets. 
Corollary 6. Suppose $G$ is compactly generated and Conditions 2 and 4 hold. Let $s_{0}$ be as in Corollary 2. Then for $A$ and $B$ in $\mathscr{B}$

$$
\lim _{n \rightarrow \infty} \frac{\mu^{\left(n+n_{0}\right)}(x+A)}{\mu^{(n)}(y+B)}=\left(g\left(s_{0}\right)\right)^{n_{0}} \exp \left(s_{0}(y-x)\right) \frac{\int_{A} \exp \left(-s_{0}(z)\right) d z}{\int_{B} \exp \left(-s_{0}(z)\right) d z}
$$

uniformly for $x$ and $y$ in compact sets.

Theorem 1 and its consequences generalize the well-known ratio limit theorem of Chung and Erdös [1] and extensions by Kemeny [3], Kesten [4], Neveu [5], Ornstein [6], and the author [9] in the Euclidean case. The proofs given here use techniques from all of these papers. In particular, some key ideas used in proving Theorem 1 were taken from Ornstein's paper.

Theorems 2 and 3 and their consequences appear to be new even in the Euclidean case. Their proofs are patterned after the method used by the author in [9].

For some extensions and applications of the above results, the reader is referred to Port and Stone [7].

\section{Proofs.}

Proof of Proposition 1. We observe first that the proposition is true if $G$ is a vector group and more generally if $G$ is the direct product of a vector group, a lattice group, a toroidal group, and a finite group. By using the structure theory for locally compact Abelian groups we will reduce the general case to this special case.

In general there is a compactly generated open and closed subgroup $H$ [2, p. 36]. It suffices to prove the lemma for the subgroup $H$. In other words, without loss of generality we can assume that $G$ is compactly generated.

Let $\varepsilon>0$ be given. Let $A$ be a compact set of positive Haar measure. There is an open set $Q$ such that $A \subset Q$ and $|Q| \leqq|A|(1+\varepsilon)^{1 / 3}$. There is an open neighborhood $U_{1}$ of $e$ such that $A+U_{1} \subset Q$.

Let $B$ be an open set having finite positive Haar measure. There is a compact set $E \subset B$ such that $(1+\varepsilon)^{1 / 3}|E|>|B|$. There is an open relatively compact set $R$ such that $E \subset R \subset \bar{R} \subset B$, where $\bar{R}$ denotes the closure of $R$. There is an open neighborhood $U_{2}$ of $e$ such that $R+U_{2} \subset B$.

Let $U=U_{1} \cap U_{2}$. There is a compact subgroup $C$ such that $C \subset U$ and $G / C$ is the direct product of a vector group, a lattice group, a toroidal group, and a finite group [2, p. 89]. Thus, by the first part of this proof there is an open set $P \subset G$, a compact set $D \subset P$, integers $m$ and $k$, and points $x_{1}, \ldots, x_{m}, y_{1}, \ldots, y_{k}$ in $G$ such that $D$ and $P$ are both unions of cosets of $C, m|R+C| \leqq(1+\varepsilon)^{1 / 3} k|A+C|$, $\bigcup_{1 \leqq j \leqq m} x_{j}+D \supset A+C$ and $y_{j}+P, 1 \leqq j \leqq k$, are disjoint subsets of $R+C$. Therefore 
$m|B| \leqq(1+\varepsilon) k|A|, \bigcup_{1 \leqq j \leqq m} x_{j}+D \supset A$, and $y_{j}+P, 1 \leqq j \leqq k$, are disjoint subsets of $B$, as desired.

LEMMA 1. Let $y$ be any point in $G$ and $U$ any open neighborhood of $e$. Then for $n$ sufficiently large there is a $z \in G$ such that $\mu^{(n)}(z+U)>0$ and $\mu^{(n)}(y+z+U)>0$.

Proof. By our basic assumption of $\S 1$ we can find a positive integer $m$ and points $s_{1}, \ldots, s_{2 m}$ in $S$ such that $s_{1}+\cdots+s_{m}-s_{m+1}-\cdots-s_{2 m} \in y+U$. Then for $n \geqq m$ we can find points $s_{1}^{\prime}, \ldots, s_{2 n}^{\prime}$ in $S$ such that $s_{1}^{\prime}+\cdots+s_{n}^{\prime}-s_{n+1}^{\prime}-\cdots-s_{2 n}^{\prime} \in y+U$. Let $z=s_{n+1}^{\prime}+\cdots+s_{2 n}^{\prime}$. Then $\mu^{(n)}(z+U)>0$. Moreover $s_{1}^{\prime}+\cdots+s_{n}^{\prime} \in y+z+U$, so that $\mu^{(n)}(y+z+U)>0$ as desired.

LEMMA 2. Let $P$ be an open set, $D$ a compact subset of $P, y \in G$, and $n_{0}$ an integer. Then for every $\varepsilon>0$ there is a $\delta>0$ such that for $n$ sufficiently large

$$
\mu^{\left(n+n_{0}\right)}(x+y+D) \leqq(1+\varepsilon) \mu^{(n)}(x+P)+e^{-\delta n}, \quad x \in G .
$$

Proof. We can find an open neighborhood $U$ of $e$ such that $D+U-U \subset P$. By Lemma 1 there is a positive integer $n_{1}$ and a $z \in G$ such that $\mu^{\left(n_{1}\right)}(z+U)>0$ and $\mu^{\left(n_{1}\right)}(y+z+U)>0$. We can write $\mu^{\left(n_{1}\right)}=a \varphi+b \chi+c \nu$ where $a, b, c$ are strictly positive constants such that $a+b+c=1$ and $\varphi, \chi, \nu$ are regular probability measures on $G, \varphi$ being concentrated on $z+U$ and $\chi$ being concentrated on $y+z+U$. Let $r$ denote a fixed integer. Then

$$
\mu^{\left(m n_{1}+r\right)}=\mu^{(r)} * \sum_{j, k} K(j, k, m) \varphi^{(j)} * \chi^{(k)} * \nu^{(m-j-k)},
$$

where $*$ denotes convolution and

$$
K(j, k, m)=\frac{m ! a^{j} b^{k} c^{m-j-k}}{j ! k !(m-j-k) !}
$$

Let $\varepsilon>0$ be given. Set

$$
A_{m}(\varepsilon)=\{(j, k) \mid 0 \leqq j, k \leqq m \text { and } K(j+1, k-1, m) \leqq(1+\varepsilon) K(j, k, m)\} .
$$

As is well known and easily shown, there is a $\delta>0$ such that for $m$ sufficiently large

$$
\sum_{A_{m}^{c}(\varepsilon)} K(j, k, m) \leqq \exp \left(\delta\left(m n_{1}+r\right)\right)
$$

where $A_{m}^{c}(\varepsilon)=\left\{(j, k) \mid 0 \leqq j, k \leqq m\right.$ and $\left.(j, k) \notin A_{m}(\varepsilon)\right\}$. Now

$$
\begin{aligned}
\sum_{A_{m}(\varepsilon)} K(j, k, m) \varphi^{(j)} * \chi^{(k)} * \nu^{(m-j-k)} & \leqq(1+\varepsilon) \sum_{A_{m}(\varepsilon)} K(j+1, k-1, m) \varphi^{(j)} * \chi^{(k)} * \nu^{(m-j-k)} \\
& \leqq(1+\varepsilon) \sum_{j \geqq 1} K(j, k, m) \varphi^{(j-1)} * \chi^{(k+1)} * \nu^{(m-j-k)}
\end{aligned}
$$


Further for $j \geqq 1$

$$
\begin{aligned}
\varphi^{(j-1)} * \chi^{(k+1)} * \nu^{(m-j-k)} * \mu^{(r)}(x+y+D) & \leqq \varphi^{(j-1)} * \chi^{(k)} * \nu^{(m-j-k)} * \mu^{(r)}(x-z+D-U) \\
& \leqq \varphi^{(j)} * \chi^{(k)} * \nu^{(m-j-k)} * \mu^{(r)}(x+D+U-U) \\
& \leqq \varphi^{(j)} * \chi^{(k)} * \nu^{(m-j-k)} * \mu^{(r)}(x+P) .
\end{aligned}
$$

Combining the above we see that for $m$ sufficiently large

$$
\mu^{\left(m n_{1}+r\right)}(x+y+D) \leqq(1+\varepsilon) \mu^{\left(m n_{1}+r\right)}(x+P)+e^{-\delta n}, \quad x \in G .
$$

By letting $r$ run through $0, \ldots, n_{1}-1$, we see that Lemma 2 holds in the special case $n_{0}=0$.

In order to complete the proof of Lemma 2 it suffices to show that there is a $\delta>0$ and $y_{0} \in G$ such that for $n$ sufficiently large

$$
\mu^{(n)}\left(x \mp y_{0}+D\right) \leqq(1+\varepsilon) \mu^{(n \pm 1)}(x+P)+e^{-\delta n}, \quad x \in G .
$$

Choose $y_{0}$ in $S$, the support of $\mu$. Let $U$ be an open neighborhood of $e$ such that $D+U \subset P$ and $D-U \subset P$. Then $\mu\left(y_{0}+U\right)>0$. We can write $\mu=a \varphi+b \chi$ where $a$ and $b$ are strictly positive constants such that $a+b=1$ and $\varphi$ and $\chi$ are regular probability measures $\varphi$ being concentrated on $y_{0}+U$. Then

$$
\mu^{(n)}=\sum_{j} K(j, n) \varphi^{(j)} * \chi^{(n-j)}
$$

where $K(j, n)=n ! a^{j} b^{n-j} / j !(n-j) !$. Set

$$
B_{n}(\varepsilon)=\{j \mid K(j, n) \leqq(1+\varepsilon) K(j+1, n+1) \text { and } K(j, n) \leqq(1+\varepsilon) K(j-1, n-1)\} .
$$

There is a $\delta>0$ such that for $n$ sufficiently large

$$
\sum_{j \notin B_{n}(\varepsilon)} K(j, n) \leqq e^{-\delta n}
$$

Thus for sufficiently large values of $n$

$$
\begin{aligned}
\mu^{(n)}\left(x-y_{0}+D\right) & \leqq \varphi * \mu^{(n)}(x+D+U) \\
& \leqq \varphi * \mu^{(n)}(x+P) \\
& \leqq e^{-\delta n}+\sum_{B_{n}(\varepsilon)} K(j, n) \varphi^{(j+1)} * \chi^{(n-j)}(x+P) \\
& \leqq e^{-\delta n}+(1+\varepsilon) \sum_{B_{n}(\varepsilon)} K(j+1, n+1) \varphi^{(j+1)} * \chi^{(n-j)}(x+P) \\
& \leqq e^{-\delta n}+(1+\varepsilon) \mu^{(n+1)}(x+P) .
\end{aligned}
$$


Also

$$
\begin{aligned}
\mu^{(n)}\left(x+y_{0}+D\right) & \leqq e^{-\delta n}+\sum_{B_{n}(\varepsilon)} K(j, n) \varphi^{(j)} * \chi^{(n-j)}\left(x+y_{0}+D\right) \\
& \leqq e^{-\delta n}+(1+\varepsilon) \sum_{B_{n}(\varepsilon)} K(j-1, n-1) \varphi^{(j)} * \chi^{(n-j)}\left(x+y_{0}+D\right) \\
& \leqq e^{-\delta n}+(1+\varepsilon) \varphi * \mu^{(n-1)}\left(x+y_{0}+D\right) \\
& \leqq e^{-\delta n}+(1+\varepsilon) \mu^{(n-1)}(x+D-U) \\
& \leqq e^{-\delta n}+(1+\varepsilon) \mu^{(n-1)}(x+P)
\end{aligned}
$$

as desired.

Proof of Theorem 1. Let $A$ be a compact set and $B$ an open set, both having finite positive Haar measure. Let an integer $n_{0}, \varepsilon>0$, and a one point set $C=\{y\}$ be given. Let $P, D, m, k$, and $x_{1}, \ldots, x_{m}, y_{1}, \ldots, y_{k}$ be as in Proposition 1. By Lemma 2 there is a $\delta>0$ such that for $n$ sufficiently large

$$
\begin{aligned}
& \mu^{\left(n+n_{0}\right)}\left(x+y+x_{i}+D\right) \leqq(1+\varepsilon) \mu^{(n)}\left(x+y_{j}+P\right)+\frac{|A| e^{-\delta n}}{m}, \\
& x \in G, \quad 1 \leqq i \leqq m, \text { and } 1 \leqq j \leqq k .
\end{aligned}
$$

Then

$\mu^{\left(n+n_{0}\right)}(x+y+A) \leqq m(1+\varepsilon) \mu^{(n)}\left(x+y_{j}+P\right)+|A| e^{-\delta n}, \quad x \in G, \quad$ and $\quad 1 \leqq j \leqq k$.

Consequently

$$
\begin{aligned}
\mu^{\left(n+n_{0}\right)}(x+y+A) /|A| & \leqq(m|B| / k|A|)(1+\varepsilon) \mu^{(n)}(x+B) /|B|+e^{-\delta n} \\
& \leqq(1+\varepsilon)^{2} \mu^{(n)}(x+B) /|B|+e^{-\delta n}, \quad x \in G .
\end{aligned}
$$

Thus Theorem 1 is true for $C$ restricted to one-point sets. It is very easy to go from here to the general case.

Proof of Corollary 1. Let $C$ be the set of Condition 1 . We can assume that $e \in C$. We can also assume that $|\partial C|=0$. For, even if not, we can use Urysohn's lemma to find an open relatively compact set $C_{1} \supset C$ such that $\left|\partial C_{1}\right|=0$ and replace $C$ by $C_{1}$.

It now follows from Theorem 1 that for every $\varepsilon>0$ there is a $\delta_{1}>0$ such that for $n$ sufficiently large

$$
\mu^{(n)}(C) /|C| \leqq(1+\varepsilon) \mu^{(n)}(-y+C) /|C|+\exp \left(-\delta_{1} n\right), \quad y \in C .
$$


Thus for any $\delta>0$ there is a positive integer $m$ such that $\mu^{(m)}(-y+C) \geqq e^{-\delta m}$ for $y \in D$. Since for $k \geqq 1$

$$
\mu^{(k m)}(C) \geqq \int_{C} \mu^{(k m-1)}(d y) \mu^{(m)}(C-y),
$$

we see that $\mu^{(k m)}(C) \geqq e^{-\delta k m}, k \geqq 1$. Theorem 1 now implies that

$$
\lim _{n \rightarrow \infty}\left(\mu^{(n)}(C)\right)^{1 / n}=1
$$

Using this result together with Theorem 1 , we see that $\lim _{n \rightarrow \infty}\left(\mu^{(n)}(A)\right)^{1 / n}=1$ for $A \in \mathscr{A}$. A final application of Theorem 1 yields Corollary 1 as desired.

Proof of Proposition 2. The proof of this, possibly known, result is quite easy. Let $\mu$ be symmetric and let $\hat{\mu}$ be its characteristic function. Then $\hat{\mu}$ is real valued. By looking at $\hat{\mu}^{2}$ if necessary, we can assume that $\hat{\mu}$ is nonnegative. We can easily find a probability density $p$ such that $p$ is continuous and symmetric and has compact support, and $\hat{p}$ is nonnegative and integrable. The Fourier inversion formula yields

$$
\int p(y) \mu^{(n)}(d y)=\int \hat{p}(\theta) \hat{\mu}^{n}(\theta) d \theta
$$

It follows easily that

$$
\lim _{n \rightarrow \infty}\left(\int p(y) \mu^{(n)}(d y)\right)^{1 / n}=1
$$

and hence that Condition 1 holds, as desired.

If $s \in \mathscr{S}$ and $g(s)<\infty$, we can define a probability measure $\mu_{s}$ on $G$ by $d \mu_{s} / d \mu=$ $(g(s))^{-1} e^{s(x)}$ or equivalently for all Borel sets $A$

$$
\mu_{s}(A)=\int_{A}(g(s))^{-1} e^{s(x)} \mu(d x)
$$

Let $\mu_{s}^{(n)}$ denote the $n$-bold convolution of $\mu_{s}$ with itself. Then for all Borel sets $A$

$$
\begin{aligned}
& \mu_{s}^{(n)}(A)=\int_{A}(g(s))^{-n} e^{s(x)} \mu^{(n)}(d x) \\
& \mu^{(n)}(A)=\int_{A}(g(s))^{n} e^{-s(x)} \mu_{s}^{(n)}(d x) .
\end{aligned}
$$

We observe that $\mu_{s}$ has the same support as $\mu$. 
Proof of Proposition 3. It follows from the above formula that if $g(s)<1$ and $A$ is compact, then

$$
\limsup _{n \rightarrow \infty}\left(\mu^{(n)}(A)\right)^{1 / n} \leqq g(s)<1,
$$

as desired.

Suppose $G$ is compactly generated. Then $G$ is the direct sum of a compact group $D$, a vector group $V$, and a lattice group $L$ [2, p. 90]. Suppose $V$ is isomorphic to $R^{a}$ and $L$ is isomorphic to $Z^{b}, Z$ denoting the set of integers. Let $d=a+b$. Then there is an isomorphism $\varphi^{\prime}$ from $V+L$ onto the subgroup of $R^{d}$

$$
G_{1}=\left\{y=\left(y_{1}, \ldots, y_{d}\right) \mid y_{a+1}, \ldots, y_{d} \text { are integers }\right\}
$$

Let $\varphi$ denote the function from $G$ to $R^{d}$ defined as follows: given $x \in G$, let $z(x)$ be the unique point in $V+L$ such that $x=z(x)+D$; let $\varphi(x)=\varphi^{\prime}(z(x))$. Then $\varphi$ is a continuous homomorphism from $G$ into $R^{d}$. Let $\mathscr{T}$ denote the dual to $R^{d}$. For $t \in \mathscr{T}$ and $y \in R^{d}$, we write $t(y)=t \cdot y$ as usual. Clearly $t \circ \varphi$ is a continuous homomorphism from $G$ to $R$ and every continuous homomorphism from $G$ to $R$ is of the form $t \circ \varphi$ for some $t \in \mathscr{T}$. Let $\nu$ be the probability measure induced on $R^{d}$ by the probability measure $\mu$ on $G$ and the map $\varphi$ from $G$ to $R^{d}$. We note that $\nu^{(n)}$, the $n$-fold convolution of $\nu$ with itself, is the measure induced on $R^{d}$ by $\mu^{(n)}$ and the $\operatorname{map} \varphi$.

Let $g_{1}$ denote the extended real-valued function defined on $\mathscr{T}$ by

$$
g_{1}(t)=\int_{R^{d}} \exp (t \cdot y) \nu(d y), \quad t \in \mathscr{T}
$$

We will have need later of Cramer's condition, which we denote as

Condition 5. ( $G$ assumed compactly generated.) There is a constant $c>0$ such that

$$
\int_{R^{d}} \exp (e|y|) \nu(d y)<\infty .
$$

Then $g_{1}|t|$ is finite and infinitely differentiable for $|t|<c$.

Proof of Proposition 4. That Condition 1 holds only if $g(s) \geqq 1$ for all $s \in \mathscr{S}$ is just Proposition 3. Conversely, suppose $G$ is compactly generated and $g(s) \geqq 1$ for all $s \in \mathscr{S}$. Then $g_{1}(t) \geqq 1$ for all $t \in \mathscr{T}$. It follows from Lemma 5 of [9] that there is a compact set $C_{1} \subset R^{d}$ such that $\lim _{n \rightarrow \infty}\left(\nu^{(n)}\left(C_{1}\right)\right)^{1 / n}=1$ and Condition 1 holds with $C=\varphi^{-1}\left(C_{1}\right)$, which is clearly compact.

Proof of Corollary 2. Suppose Condition 2 holds. Then $\nu\{y \mid t \cdot y>0\}>0$ for all $t \in \mathscr{T}$ except $t=0$; i.e., in the terminology of [9], $\nu$ is not one-sided. Thus as in [9] there is a unique $t_{0} \in \mathscr{T}$ such that $g_{1}\left(t_{0}\right) \leqq g_{1}(t)$ for $t \in \mathscr{T}$. Define $s_{0} \in \mathscr{S}$ by 
$s_{0}=t_{0} \circ \varphi$. Then $s_{0}$ is the unique point in $\mathscr{S}$ such that $g\left(s_{0}\right) \leqq g(s), s \in \mathscr{S}$. From Proposition 4 and Corollary 1 we see that for any integer $n_{0}$ and $A$ and $B$ in $\mathscr{A}$

$$
\lim _{n \rightarrow \infty} \frac{\mu_{s_{0}}^{\left(n+n_{0}\right)}(x+A)}{\mu_{s_{0}}^{(n)}(y+B)}=\frac{|A|}{|B|}
$$

uniformly for $x$ and $y$ in compact sets. By partitioning $A$ and $B$ into a finite number of sets in $\mathscr{A}$ on which $\exp \left(-s_{0}(x)\right)$ is approximately a constant, we get the conclusion of Corollary 2, as desired.

Suppose $\mu^{(n)}$ is absolutely continuous and has a continuous and bounded density $p^{(n)}$ with respect to Haar measure on $G$. Then $\nu^{(n)}$ is absolutely continuous and has a continuous and bounded density $q^{(n)}$ with respect to Haar measure on $G_{1}$.

Lemma 3. Suppose $G$ is compactly generated and Condition 3 holds. Then there are constants $a>0$ and $\delta>0$ such that for $n$ sufficiently large

$$
\left|p^{(n)}(x)-a q^{(n)}(\varphi(x))\right| \leqq e^{-\delta n}, \quad x \in G .
$$

Proof of Lemma 3. If $\theta$ is a character of $G$, write $\theta(x)=(\theta, x)$. Then for $n$ sufficiently large $\hat{\mu}^{n}$ is integrable and

$$
\begin{aligned}
p^{(n)}(x) & =\int(\theta, x) \hat{\mu}^{n}(\theta) d \theta \\
& =\iint\left(\theta_{1}, x\right)\left(\theta_{2}, x\right) \hat{\mu}^{n}\left(\theta_{1}, \theta_{2}\right) d \theta, d \theta_{2}
\end{aligned}
$$

where $\theta_{1}$ and $\theta_{2}$ denote arbitrary elements in the dual groups of $V+L$ and $D$ respectively. Observe that $\hat{\mu}$ is continuous and is less than 1 in absolute value except when $\theta=0$. Since the dual of $D$ is discrete, there is a $\delta>0$ such that for $n$ sufficiently large (see [8, Theorem 2.6.6])

$$
\int_{\theta_{2} \neq 0}\left|\hat{\mu}\left(\theta_{1}, \theta_{2}\right)\right|^{n} d \theta, d \theta_{2} \leqq e^{-2 \delta n} .
$$

Thus there is a constant $a>0$ such that for $n$ sufficiently large

$$
\left|p^{(n)}(x)-a_{1} \int\left(\theta_{1}, x\right) \hat{\mu}^{n}\left(\theta_{1}, 0\right) d \theta_{1}\right| \leqq e^{-\delta n}, \quad x \in G
$$

There is also a constant $a_{2}>0$ such that

$$
\int\left(\theta_{1}, x\right) \hat{\mu}^{n}\left(\theta_{1}, 0\right) d \theta_{1}=a_{2} q^{(n)}(\varphi(x))
$$

By setting $a=a_{1} a_{2}$, we see that Lemma 3 holds, as desired. 
LemMa 4. Suppose $G$ is compactly generated and Conditions 3 and 5 hold. Let $m_{0}$ denote the mean of $\nu$. Then for every $\tau>0$ there is $a \delta>0$ such that for $n$ sufficiently large

$$
q^{(n)}(y) \leqq e^{-\delta n}, \quad y \in G_{1} \text {, and }\left|y-n m_{0}\right|>\tau n .
$$

Proof of Lemma 4. By Condition 3 we can obtain an integer $n_{0}$ and a positive constant $M<\infty$ such that $\nu^{\left(n_{0}\right)}$ is absolutely continuous with respect to Haar measure on $G_{1}$ and has a density $q^{\left(n_{0}\right)}$ such that $q^{\left(n_{0}\right)}(y) \leqq M, y \in G_{1}$. Then for $n$ sufficiently large $\nu^{(n)}$ is absolutely continuous and has a continuous density $q^{(n)}$ such that $q^{(n)}(y) \leqq M, y \in G_{1}$, and

$$
q^{(n)}(y)=\int q^{\left(n_{0}\right)}(z) q^{\left(n-n_{0}\right)}(y-z) d z, \quad y \in G_{1} .
$$

Choose $\tau>0$. Since $\nu$ satisfies Cramer's condition there is (see [9]) a $\delta>0$ such that for $n$ sufficiently large

$$
\nu^{\left(n-n_{0}\right)}\left\{y|| y-n m_{0} \mid \geqq \tau n / 2\right\} \leqq e^{-\delta n} / 2 M,
$$

and

$$
\nu^{\left(n_{0}\right)}\{y|| y \mid \geqq \tau n / 2\} \leqq e^{-\delta n} / 2 M .
$$

Then for $n$ sufficiently large, we have that for $\left|y-m_{0} n\right| \geqq \tau n$

$$
\begin{aligned}
q^{(n)}(y) \leqq & \int_{|z| \geqq n n / 2} q^{\left(n_{0}\right)}(z) q^{\left(n-n_{0}\right)}(y-z) d z \\
& \quad+\int_{\left|y-z-n m_{0}\right| \geqq \imath n / 2} q^{\left(n_{0}\right)}(z) q^{\left(n-n_{0}\right)}(y-z) d z \leqq e^{-\delta n},
\end{aligned}
$$

as desired.

We assume now that the Haar measure on $G_{1}$ is chosen so that the Haar measure of

$$
\left\{\left(y_{1}, \ldots, y_{d}\right)|| y_{1}|\leqq 1 / 2, \ldots,| y_{a} \mid \leqq 1 / 2, y_{a+1}=\cdots=y_{d}=0\right\}
$$

is equal to 1 .

Let $\nu_{t}$ be defined in terms of $\nu$ in the same way that $\mu_{s}$ is defined in terms of $s$. Let $m_{t}$, and $\Sigma_{t}$ denote the mean and covariance of $\nu_{t}$. The functions $m_{t}$ and $\Sigma_{t}$ are continuous and infinitely differentiable for $|t|<c$. Also, as $t \rightarrow 0$

$$
m_{t}=m_{0}+\Sigma_{0} t+O\left(|t|^{2}\right) \quad \text { and } \quad \Sigma_{t}=\Sigma_{0}+O(|t|)
$$

Let $T$ denote the support of $\nu$. Then $G_{1}$ is the smallest closed group containing $T-T$. Thus, in particular, $\Sigma_{0}$ is nondegenerate and $m_{t}$ has an inverse $t_{m}$ for $m-m_{0}$ sufficiently small, which is infinitely differentiable. 
Lemma 5. Suppose $G$ is compactly generated and Conditions 3 and 5 hold. Then for some constant $\tau_{1}>0$

$$
q^{(n)}(y)=\left(g\left(t_{y / n}\right)\right)^{n} \exp \left(-y \cdot t_{y / n}\right)\left((2 \pi n)^{d}\left|\Sigma_{t_{y / n}}\right|\right)^{-1 / 2}\left(\left(1+o_{n}(1)\right), \quad y \in G_{1},\right.
$$

where $o_{n}(1) \rightarrow 0$ as $n \rightarrow \infty$ uniformly for $\left|y-n m_{0}\right| \leqq \tau_{1} n$.

The proof of this lemma is quite similar to the proof of Theorem 3 of [9] and will be omitted.

LEMMA 6. Theorem 2 holds under the restrictions that $G$ be compactly generated and that Condition 5 holds.

Proof of Lemma 6. It follows from Lemmas 4 and 5 that for any compact set $C \subset G$ integer $n_{0}$ and $\varepsilon>0$, there is a $\delta>0$ such that for $n$ sufficiently large

$$
q^{\left(n+n_{0}\right)}(\varphi(x+y)) \leqq(1+\varepsilon) q^{(n)}(\varphi(x))+e^{-\delta n}, \quad x \in G \quad \text { and } \quad y \in C,
$$

and the conclusion of Theorem 2 now follows from Lemma 3.

Proof of Theorem 2. It follows from Condition 3 that there is a positive constant $M<\infty$ such that for $n \geqq n_{1} \mu^{(n)}$ is absolutely continuous with respect to Haar measure and has a continuous density $p^{(n)} \leqq M$. In proving Theorem 2 it suffices to consider integers $n_{0}= \pm n_{2}$, where $n_{2} \geqq n_{1}$. Given such an $n_{2}$ we can write $\mu^{\left(n_{2}\right)}=$ $(\varphi+\chi) / 2$ where $\varphi$ and $\chi$ are regular probability measures having continuous bounded densities, $\varphi$ having compact support. Let $C$ be a compact subset of $G$. Then (see [2, p. 36]) we can find an open and closed compactly generated subgroup $H$ of $G$ such that $C \subset H$ and $\varphi(H)=1$. From Lemma 6 we see that for every $\varepsilon>0$ there is a $\delta>0$ such that for $n$ sufficiently large

$$
(1+\varepsilon)^{1 / 2} p_{\varphi}^{(n)}(x+y) \leqq(1+\varepsilon) p_{\varphi}^{(n \pm 1)}(x)+e^{-4 \delta n} / 2, \quad x \in H \quad \text { and } \quad y \in C,
$$

where $p_{\varphi}^{(n)}$ denotes the continuous bounded density of $\varphi$. For fixed $r$

$$
\mu^{\left(r+n n_{2}\right)}=\mu^{(r)} * \sum_{j} K(j, n) \varphi^{(j)} * \chi^{(n-j)}
$$

where $K(j, n)=n ! / 2^{n} j !(n-j) !$. Set

$$
\begin{aligned}
B_{n}(\varepsilon)=\left\{j \mid n / 4 \leqq j<n, K(j, n) \leqq(1+\varepsilon)^{1 / 2} K(j+1, n+1)\right. \\
\text { and } \left.K(j, n) \leqq(1+\varepsilon)^{1 / 2} K(j-1, n-1)\right\}
\end{aligned}
$$

By making $\delta$ smaller if necessary, but still positive, we have that for $n$ sufficiently large

$$
\sum_{j \notin B_{n}(\varepsilon)} K(j, n) \leqq e^{-\delta n} / 2 M
$$


Thus for $n$ sufficiently large

$$
\mu^{(r)} * \sum_{j \notin B_{n}(\varepsilon)} K(j, n) \varphi^{(j)} * \chi^{(n-j)}
$$

has a density bounded above by $e^{-\delta n} / 2$. Also the density of

$$
\mu^{(r)} * \sum_{j \in B_{n}(\varepsilon)} K(j, n) \varphi^{(j)} * \chi^{(n-j)}
$$

at $x+y$ is bounded above by

$$
(1+\varepsilon) p^{\left(r+n n_{2} \pm n_{2}\right)}(x)+e^{-\delta n} / 2 .
$$

In summary

$$
p^{\left(r+n n_{2}\right)}(x+y) \leqq(1+\varepsilon) p^{\left(r+n n_{2} \pm n_{2}\right)}(x)+e^{-\delta n}, \quad x \in G \quad \text { and } \quad y \in C
$$

from which Theorem 2 easily follows.

Proof of Corollary 4. Let $s_{0}$ be as in Corollary 2. Then by Proposition 4 the measures $\mu_{s_{0}}^{(n)}$ satisfy Condition 1 . Let $p_{s_{0}}^{(n)}$ be their densities. For $n$ sufficiently large

$$
p_{s 0}^{(n)}(x)=\left(g\left(s_{0}\right)\right)^{-n} e^{s(x)} p^{(n)}(x)
$$

so that while the densities $p_{s_{0}}^{(n)}$ are continuous they are not necessarily bounded and Theorem 2 is not directly applicable (this made a gap in an earlier proof of Corollary 4 which was found by the referee). However, the proof of Theorem 2 can be modified to yield Corollary 4 . We can write $\mu_{s_{0}}^{\left(n_{2}\right)}=(\varphi+\chi) / 2$, where $\varphi$ is a regular probability measure having compact support and a continuous bounded density, and $\chi$ is a regular probability measure which has a continuous density $p_{x}$ such that $\exp \left(-s_{0}(x)\right) p_{x}(x)$ is bounded and integrable.

The only point where the proof of Theorem 4 breaks down is the estimate of the density of

$$
\mu_{s_{0}}^{(r)} * K(O, n) \chi^{(n)}=\frac{1}{2^{n}} \mu_{s_{0}}^{(r)} * \chi^{(n)}
$$

We can assume that $r$ is large enough so that $\mu_{s_{0}}^{(r)}$ is absolutely continuous and has a continuous density which is bounded above by $M \exp \left(s_{0}(x)\right)$ for some finite number $M$ which may depend on $r$ but not on $x$. We need

LEMMA 7. Let $\chi$ be a regular probability measure on $G$ which is absolutely continuous and has a continuous density $p_{x}$ such that, for some $M<\infty, p_{x}(x) \leqq$ $M \exp \left(s_{0}(x)\right)$ for $x \in G$. Then for $n \geqq 1 \chi^{(n)}$ is absolutely continuous and has $a$ continuous density $p_{x}^{(n)}$ such that $p_{x}^{(n)}(x) \leqq M \exp \left(s_{0}(x) / n\right)$ for $x \in G$. 
Proof. The proof proceeds by induction. Assume the lemma is true for $n$. Then

$$
\left(p_{x}^{(n)}(y)\right)^{n / n+1}\left(p_{x}(x-y)\right)^{1 / n+1} \leqq M \exp \left(s_{0}(x) / n+1\right)
$$

and hence

$$
p_{x}^{(n)}(y) p_{x}(x-y) \leqq M \exp \left(s_{0}(x) / n+1\right)\left(p_{x}^{(n)}(y)\right)^{1 / n+1}\left(p_{x}(x-y)\right)^{n / n+1} .
$$

By Hölder's inequality

$$
\int_{G}\left(p_{x}^{(n)}(y)\right)^{1 / n+1}\left(p_{x}(x-y)\right)^{n / n+1} d y \leqq 1 .
$$

Thus the boundedness property holds for $n+1$. The continuity property follows easily as well. This completes the proof of Lemma 7 .

Let $q$ denote the density of $\mu_{s_{0}}^{(r)}$ and suppose that $q(x) \leqq M \exp \left(s_{0}(x)\right)$ for $x \in G$ and $p_{x}^{(n)}(x) \leqq M \exp \left(s_{0}(x) / n\right)$ for $x \in G$ and $n \geqq 1$. Then by the same argument $2^{-n} \mu_{s_{0}}^{(r)} * \chi^{(n)}$ has a density bounded above by $2^{-n} M \exp \left(s_{0}(x) / n+1\right)$. Using this estimate we get something slightly weaker than the conclusion of Theorem 2 : for any compact set $C$, integer $n_{0}$ and $\varepsilon>0$, there is a $\delta>0$ such that for $n$ sufficiently large

$$
p_{s_{0}}^{\left(n+n_{0}\right)}(x+y) \leqq(1+\varepsilon) p_{s_{0}}^{(n)}(x)+e^{-\delta n}, \quad x, y \in C .
$$

Since the measures $\mu_{s_{0}}^{(n)}$ satisfy Condition 1, it follows that

$$
\lim _{n \rightarrow \infty} \frac{p_{s_{0}}^{\left(n+n_{0}\right)}(x+y)}{p_{s_{0}}^{(n)}(x)}=1
$$

uniformly for $x$ and $y$ in compact sets. But

$$
p^{(n)}(x)=\left(g\left(s_{0}\right)\right)^{n} \exp \left(-s_{0}(x)\right) p_{s_{0}}^{(n)}(x),
$$

and Corollary 4 now follows immediately.

Proof of Theorem 3. There is an integer $n$ such that $\mu^{\left(n_{1}\right)}=(\varphi+\chi) / 2$ where $\varphi$ and $\chi$ are regular probability measures, $\varphi$ being absolutely continuous and having a continuous bounded density. The proof of Theorem 3 now continues along the lines of the proof of Theorem 2, using Theorem 2 instead of Lemma 6.

Corollary 5 follows immediately from Theorem 3. Corollary 6 follows immediately once we verify that for any integer $n_{0}$ and sets $A$ and $B$ in $\mathscr{B}$

$$
\lim _{n \rightarrow \infty} \frac{\mu_{s_{0}}^{\left(n+n_{0}\right)}(x+A)}{\mu_{s_{0}}^{(n)}(y+B)}=\frac{|A|}{|B|}
$$

uniformly for $x$ and $y$ in compact sets. But this result follows from Proposition 4 and Corollary 5. 


\section{REFERENCES}

1. K. L. Chung and P. Erdös, Probability limit theorems assuming only the first moment, Mem. Amer. Math. Soc., No. 6 (1951), 19 pp.

2. E. Hewitt and K. A. Ross, Abstract harmonic analysis, Academic Press, New York, 1963.

3. J. G. Kemeny, A probability limit theorem requiring no moments, Proc. Amer. Math. Soc. 10 (1959), 607-612.

4. H. Kesten, Ratio limit theorems for random walks. II, J. Analyse Math. 9 (1963), 323-379.

5. J. Neveu, Sur le théorème ergodique de Chung-Erdös, C. R. Acad. Sci. Paris 257 (1963), 2953-2955.

6. D. Ornstein, A limit theorem for independent random variables, (unpublished).

7. S. Port and C. J. Stone, Limit theorems on hitting times for random walks on groups, (to appear).

8. W. Rudin, Fourier analysis on groups, Interscience, New York, 1962.

9. C. J. Stone, Local and ratio limit theorems, Proc. Fifth Berkeley Sympos. on Prob. and Stat., (to appear).

UNIVERSITY OF CALIFORNIA, Los ANGeles, California 